\title{
Una reflexión sobre la labor social en pacientes con trastorno antisocial de la personalidad
}

\author{
A Reflection on Social Work in Patients with Antisocial Personality \\ Disorder
}

\section{Uma reflexão sobre o trabalho social com pacientes vitimas do transtorno de personalidade anti-social}

Ana Liseth Herrera-Gómez, MD. *

\section{Resumen}

Introducción: El trastorno antisocial de la personalidad está vinculado a la clasificación de trastornos de la personalidad en la salud mental. Este diagnóstico se les otorga a personas que presenten rasgos de personalidad específicos e inflexibles que afectan su funcionalidad y en los que no se encuentra otra causa médica aparente. Existen diversos tratamientos posibles para estos pacientes y cuyo manejo debe ser individualizado según su edad y progresión de los rasgos de personalidad. Este trastorno genera un impacto adicional a la comunidad debido a su relación con conductas delictivas e inapropiadas de los pacientes con este diagnóstico, convirtiéndolo en un asunto de interés para la convivencia social. Objetivo: Reflexionar acerca del rol de la sociedad en la prevención, inclusión y manejo de pacientes con trastorno antisocial de la personalidad. Discusión: Conociendo las variables involucradas en el desarrollo de trastornos de personalidad como el antisocial, además del impacto social que genera la expresión de dichos síntomas en la comunidad, se hace necesario una intervención multidisciplinaria desde la sociedad y sectores públicos como salud y justicia a la hora de abordar estos pacientes, pues medidas como mejorar el ambiente que los rodea y el reconocimiento a temprana edad mejora los resultados en el tratamiento y podría disminuir la expresión de la misma, así como las consecuencias sociales producto de casos graves. Conclusiones: El trastorno antisocial de la personalidad es un diagnóstico psiquiátrico que involucra necesariamente al paciente y la comunidad. La concientización de esta patología en la sociedad podría dar herramientas para mejorar la prevención, diagnóstico y atención integral con resultados a nivel individual y social. [Herrera-Gómez AL. Una reflexión sobre la labor social en pacientes con trastorno antisocial de la personalidad. MedUNAB 2017-2018; 20(3): 368-373].

Palabras clave: Trastorno de Personalidad Antisocial; Diagnóstico; Psiquiatría; Psiquiatría Comunitaria; Empatía; Participación de la Comunidad.

\footnotetext{
* Médica y Cirujana, Universidad Industrial de Santander, Bucaramanga, Santander, Colombia.

Correspondencia: Ana Liseth Herrera-Gómez. Calle 18 No. 13-25, Barrio Gaitán, Bucaramanga, Santander, Colombia. E-mail: ana7hg@gmail.com 


\section{Abstract}

Introduction: The antisocial personality disorder is linked to the classification of personality disorders in mental health. This diagnosis is given to people who have specific and inflexible personality traits that affect their functionality and in which no other apparent medical cause is found. There are several possible treatments for these patients and their management must be individualized according to their age and the progression of their personality traits. This disorder generates an additional impact on the community due to its relationship with criminal and inappropriate behavior of patients with this diagnosis, making it a matter of interest for social coexistence. Objective: To reflect on the role of society in the prevention, inclusion and management of patients with antisocial personality disorder. Discussion: By knowing the variables involved in the development of personality disorders such as antisocial, besides the social impact generated by the expression of these symptoms in the community, it is necessary a multidisciplinary intervention from society and public sectors such as health and justice when dealing with these patients. Furthermore, if some measures are adopted such as improving the environment that surrounds patients and the recognition at an early age of this disorder, these measures could improve the results in the treatment and reduce the expression of itself, as well as the social consequences of serious cases of this disorder. Conclusions: The antisocial personality disorder is a psychiatric diagnosis that involves the patient and community. The awareness of this pathology in society could provide tools to improve prevention, diagnosis and comprehensive care with individual and social results. [Herrera-Gómez AL. A Reflection on Social Work in Patients with Antisocial Personality Disorder. MedUNAB 2017-2018; 20(3): 368-373]

Keywords: Antisocial Personality Disorder; Diagnosis; Psychiatry; Community Psychiatry; Empathy; Community Participation.

\section{Introducción}

Los trastornos de personalidad hacen parte de un sección especial de la psiquiatría que clasifica rasgos de personalidad que pueden estar presentes causando algún malestar o deterioro social, asimismo estos rasgos inflexibles no pueden ser ocasionados por otra causa mental u orgánica; tales son, entonces, las características que configuran un trastorno de personalidad $(1,2)$.

Dentro de los diez trastornos de la personalidad catalogados por el Manual Diagnóstico y Estadístico de los Trastornos Mentales (DSM-5) se encuentran 3 grupos de clasificación (2). Se estima que de un 6 a $10 \%$ de la población general cumple con criterios para alguno de los trastornos de personalidad(3). Según la Organización Panamericana de la Salud en América Latina no hay suficientes cifras epidemiológicas de estos trastornos de personalidad debido a la dificultad para obtener estudios de calidad en todos los países $(4,5)$. En el caso de Colombia, según la encuesta de

\section{Resumo}

Introdução: $O$ transtorno de personalidade anti-social está vinculado à classificação de transtornos de personalidade em saúde mental. Este diagnóstico é dado a pessoas que possuem traços de personalidade específicos e inflexíveis que afetam sua funcionalidade e que não apresentam outra causa médica. Existem vários tratamentos possíveis para esses pacientes e seu cuidado deve ser individualizado de acordo com sua idade e à progressão dos traços de sua personalidade. Esta doença gera um impacto adicional na comunidade devido à sua relação com os comportamentos criminosos e inadequados dos pacientes com esse diagnóstico, tornando-se uma questão importante para a convivência social. Objetivo: Refletir sobre o papel da sociedade na prevenção, inclusão e tratamento de pacientes com transtorno de personalidade anti-social. Discussão: Conhecer as variáveis implicadas no desenvolvimento dos distúrbios da personalidade, identificada como anti-social, além do impacto social gerado pela expressão desses sintomas na comunidade, é necessária uma intervenção multidisciplinaria que envolva tanto a sociedade como os setores públicos, isto é: a saúde e a justiça no momento de abordar esses pacientes. Já que as medidas para melhorar o ambiente que os rodeia e o diagnostico a temprana idade, melhora os resultados no tratamento e pode reduzir a expressão do mesmo, bem como as conseqüências sociais dos casos graves. Conclusões: $O$ transtorno de personalidade antissocial é um diagnóstico psiquiátrico que envolve necessariamente o paciente e a comunidade. A consciência desta patologia na sociedade poderia fornecer ferramentas para melhorar a prevenção, o diagnóstico e o atendimento integral, obtendo melhores resultados individuais e sociais. [Herrera-Gómez AL. Uma reflexão sobre o trabalho social com pacientes vitimas do transtorno de personalidade anti-social. MedUNAB 20172018; 20(3): 368-373].

Palavras-chave: Transtorno da Personalidade Antissocial; Diagnóstico; Psiquiatria; Psiquiatria Comunitária; Empatia; Participação da Comunidade.

salud mental de 2015 , existe $1.8 \%$ de la población que presenta hasta 6 rasgos de personalidad antisocial, la mayoría, hombres $(6,7)$.

El trastorno antisocial de la personalidad (TAP) se ha relacionado con conductas sociales inapropiadas o delictivas (8), que requieren una atención médica y judicial. En países de América Latina se ha establecido relación con rasgos de personalidad antisocial, narcisista y obsesiva-compulsiva con casos de violencia intrafamiliar (9). Además, se ha encontrado asociación de rasgos de personalidad con criminalidad, violencia interpersonal, abuso de sustancias entre otros comportamientos destructivos (10).

El objetivo a desarrollar se centra en reflexionar acerca de la importancia en la concientización de patologías como TAP en la sociedad, pues la creación de espacios para la prevención, inclusión y manejo de estos pacientes garantiza evitar la expresión de conductas que pongan en riesgo al paciente y la comunidad que habitan. 


\section{Generalidades}

Los trastornos de la personalidad son un diagnóstico otorgado a pacientes con comportamientos y pensamientos inflexibles arraigados desde la infancia, que causan deterioro social y que no se explican por otra razón (1). Los trastornos de personalidad se clasifican en 3 grupos (2):

- Grupo A: Trastorno paranoide de la personalidad, trastorno esquizoide de la personalidad y trastorno esquizotípico de la personalidad (2).

- Grupo B: Trastorno antisocial de la personalidad, trastorno límite de la personalidad, trastorno histriónico de la personalidad y trastorno narcisista de la personalidad (2).

- Grupo C: Trastorno de la personalidad por evitación, trastorno de la personalidad por dependencia, trastorno obsesivo-compulsivo de la personalidad y el trastorno de la personalidad no especificado (2).

En cuanto al origen etiológico del TAP se ha estudiado las posibles consecuencias asociadas al embarazo como el bajo peso al nacer, el consumo de tabaco durante la gestación y pertenecer a un estrato socioeconómico bajo como factores influyentes de rasgos de comportamiento alterado en la niñez, así como también trastorno por déficit de atención e hiperactividad, encontrando una intensificación de los síntomas en pacientes con el diagnóstico y dichas exposiciones (11).

Desde el estudio de la anatomía se ha observado el papel de la amígdala como reguladora de la respuesta al miedo o estímulos emocionales, encontrando una baja reactividad de la misma a situaciones de tipo emocional en pacientes con TAP (12). Por otro lado, la genética ha buscado genes específicos que orienten al desarrollo de personalidad antisocial, sin embargo, se ha evidenciado que no existe un gen particular, sino que puede ser secundario a la expresión o no de diversos genes que estimulen un nexo a otros comportamientos como el consumo de alcohol en pacientes con rasgos antisociales (13), y hasta ahora se considera que no podría existir una relación que anticipe el diagnóstico de trastornos antisociales por estudios genéticos, pues si bien se ha encontrado genes estrechamente asociados a comportamientos antisociales como los que codifican para trasportadores de serotonina y monoamino oxidasa (14) se concluye que hay factores ambientales que influyen en el desarrollo final del trastorno (15), como maltrato infantil o negligencia por parte de los cuidadores de los pacientes en edades pediátricas (16).

Según Sigmund Freud en su teoría psicodinámica del "yo" "ello" y "super yo", el TAP se explica por un "super yo" débil que quita la responsabilidad social o la "conciencia" a estos individuos, dejando libre los impulsos del "ello" (17). Por otro lado, desde las teorías conductuales, se piensa que en pacientes con TAP no existe un aprendizaje emocional, por lo cual no responden a estímulos o situaciones con carga emocional(18).

\section{Diagnóstico}

Para la mayoría de diagnósticos en psiquiatría actualmente se utiliza la guía del DSM-5 para señalar criterios diagnósticos y objetivar la evaluación de los pacientes, a continuación se enuncia los criterios diagnósticos específicos para el TAP:

A. Patrón dominante de inatención y vulneración de los derechos de los demás, que se produce desde los 15 años de edad, y que se manifiesta por 3 o más de los hechos siguientes:

1. Incumplimiento de las normas sociales respecto a los comportamientos legales, que se manifiesta por actuaciones repetidas que son motivo de detención.

2. Engaño que se manifiesta por mentiras repetidas, utilización de alias o estafa para provecho y placer personal.

3. Impulsividad o fracaso para planificar con antelación.

4. Irritabilidad o agresividad, que se manifiesta por peleas o agresiones físicas repetidas.

5. Desatención imprudente de la seguridad propia o de los demás.

6. Irresponsabilidad constante, que se manifiesta por la incapacidad repetida de mantener un comportamiento laboral coherente o cumplir con las obligaciones económicas.

7. Ausencia de remordimientos, que se manifiesta con indiferencia o racionalización del hecho de haber herido, maltratado o robado a alguien.

B. El individuo tiene como mínimo 18 años.

C. Existen evidencias de la presencia de un trastorno de la conducta con inicio antes de los 15 años.

D. El comportamiento antisocial no aparece exclusivamente en el transcurso de una esquizofrenia o de un trastorno bipolar(2).

\section{Tratamiento}

El manejo de TAP podría ofrecerse a dos grandes grupos etarios: adultos y población infantil. Según la Asociación Española de Psiquiatría del Niño y el Adolescente (AEPNYA) el manejo temprano en niños con comportamientos antisociales se debe basar en psicoterapia que enseña valores y códigos de convivencia social utilizando reforzamiento positivo, manejo de la ira y terapias familiares para fortalecer el soporte terapéutico (19). Estas medidas buscan detener comportamientos inadecuados para evitar la consolidación de un trastorno antisocial de la personalidad en la vida adulta (16).

En un adulto con diagnóstico establecido el abordaje puede ser tardío dado la pobre introspección del paciente, como medidas psicoterapéuticas se ha planteado el uso de terapias de mentalización, que buscan enseñar al paciente a 
identificar estados mentales o emocionales en sí mismo y en otras personas (20).

Farmacológicamente se ha probado el uso de antipsicóticos atípicos como la Olanzapina partiendo del efecto en pacientes violentos con esquizofrenia en los que disminuye episodios de agresiones (21) y la Clozapina en paciente con TAP y episodios de violencia (22).

\section{Riesgo social de pacientes con TAP}

Algunas patologías psiquiátricas condicionan a estados de agitación o violencia consideradas un peligro para el paciente y la sociedad; se ha encontrado relación entre pacientes con rasgos de personalidad antisocial y el grado de violencia o criminalidad que acompaña un estado de agitación de otros trastornos psiquiátricos (23).

Si bien no se generaliza a todos los TAP con criminales, algunos de ellos son conocidos por sus actos violentos y crímenes de alta magnitud, por ejemplo el caso de Theodore Bundy, un famoso asesino serial norteamericano a quien la Asociación Americana de Psicología describió como el mejor ejemplo de TAP, fue encontrado culpable de al menos 23 asesinatos confesados utilizando manipulación y nulos sentimientos de culpa, sentenciado a muerte por la silla eléctrica el 24 de Enero de 1989 (24). A nivel nacional, se encuentra el caso de Luis Alfredo Garavito conocido como "el Monstruo de los Andes" acusado por al menos 172 crímenes de abuso sexual, tortura y asesinato a menores de edad, quien en su infancia padeció de maltrato por parte de su padre y que por leyes colombianas pueda ser dado en libertad por completar su condena a pesar de la gravedad de sus delitos (25).

\section{Rol de la comunidad en el manejo social de paciente con TAP}

Como se ha mencionado anteriormente existen tratamientos para pacientes con TAP que si bien no son definitivos buscan mejorar el estado clínico y prevenir consecuencias sociales al entorno de los pacientes (20). El papel que desempeña la comunidad con estos casos consiste en intervenir en la prevención y el tratamiento propiamente dicho del trastorno. Se ha mencionado que, a pesar de predisposiciones biológicas, los factores ambientales juegan un papel fundamental en el desarrollo y severidad de un TAP, como el hecho de la exposición a maltrato intrafamiliar o bajo estrato socioeconómico (11), así como la atención oportuna en pacientes pediátricos buscando evitar la perpetuidad de estructuras mentales antisociales que pudieran condicionar las conductas violentas o criminales en la vida adulta (19).

No obstante, es importante resaltar que no todos los pacientes presentan el riesgo de cometer actos delictivos y por tanto el manejo y la inclusión social varían de persona a persona. En este sentido, existen escalas para clasificar la severidad de trastornos de personalidad como: The Personality Inventory for DSM-5 (PID-5), Minnesota Multiphasic Personality Inventory-2-RF (MMPI-2-RF), Inventario Clínico Mutiaxial de Millon, entre otras (26). Así por ejemplo, un paciente con un historial incipiente de delincuencia, sin dependencia a sustancias y una buena red de apoyo tiene mejor pronóstico que un paciente con múltiples crímenes cometidos, consumo de sustancias psicoactivas o comorbilidad con otros trastornos mentales (27). Es decir, ejemplos mencionados como los de Ted Bundy y Luis Alfredo Garavito tienen un pronóstico nulo, en esos casos se podría afirmar que todo intento de tratamiento sería poco efectivo pues la estructura mental de estos personajes es incompatible con la sociedad actual resultando en un intento de integración fallido y peligroso, dejando como manejo más importante la intervención judicial para protección de la sociedad.

\section{Conclusiones}

Teniendo en cuenta lo conocido de la patología, las posibilidades terapéuticas y el riesgo social con algunos de los pacientes con TAP, reflexionar acerca de cuál debería ser la postura como sociedad frente a estos pacientes permite anticipar problemáticas futuras. Hasta ahora, la acción social solo actúa judicialmente para una persona que comete delitos, siendo judicializados y sentenciados por los crímenes cometidos. Pero si se considera que algunos de estos delitos son cometidos por personas que pueden encontrarse en un diagnóstico médico de TAP, se podría llegar a pensar que de haber existido una buena red de apoyo, una atención médica oportuna que detectara rasgos de comportamiento inadecuado en la niñez y que con esto se iniciara un tratamiento médico y multidisciplinario temprano, muy probablemente varios crímenes no se llevarían a cabo.

Desde el punto de vista preventivo, como sociedad se debe reconocer la oportunidad que se tiene de captar y atender las necesidad de pacientes con riesgo de TAP, no solo por el derecho a la atención médica sino por las consecuencias para el paciente y la sociedad que habita si se deja avanzar una patología a la que se le asocian conductas peligrosas o delictivas, y a su vez priorizar la necesidad de minimizar los factores asociados como embarazos con riesgo biopsicosocial, violencia en la infancia, negligencia, bajo nivel educativo o socioeconómico.

Por otro lado, el tratamiento de pacientes con diagnóstico ya establecido debe individualizarse. La controversia radica en los casos extremos de pacientes con crímenes mayores, en los que son riesgo para la sociedad y se sabe de las bajas probabilidades de mejoría clínica. En estos casos la Medicina debe actuar en conjunto con la Ley, y así la reflexión se traslada a cada escenario y tipo de civilizaciones posibles en 
el mundo, sociedades como la estadounidense resuelve casos graves de TAP mediante la Ley, que dicta prisión perpetua o pena de muerte de criminales que hayan cometido delitos graves. Por otro lado, en sociedades como la latinoamericana asesinos seriales son condenados a años de prisión con la posibilidad de salir libres y de que podrían reincidir en sus actos delictivos. Formas de acción tan diferentes entre países pueden ser influenciadas por el nivel de desarrollo, las bases de leyes en el Estado, el papel de la Iglesia en la sociedad y el nivel de tolerancia de la comunidad.

En conclusión, el fin de este ensayo no es encontrar una respuesta universal al correcto proceder legal y médico de la sociedad ante pacientes con TAP, puesto que cada caso es particular y, en este sentido, se vuelve complejo formular una conclusión general al respecto. La finalidad es dar un punto de partida para la reflexión de estos casos y lograr un mejor entendimiento de la importancia de la prevención de enfermedades médicas en general. Específicamente, en el caso de TAP garantizar un entorno adecuado para la sociedad, lograr identificar un niño con rasgos antisociales y otorgarle un manejo temprano disminuye el riesgo de exposición para la patología del paciente y con esto un menor riesgo de conflictos sociales para la comunidad.

\section{Conflicto de intereses}

\section{La autora declara no tener conflicto de interés.}

\section{Referencias}

1. Wang, Y. Et al. Screening cluster A and cluster B personality disorders in Chinese high school students. BMC Psychiatry [internet]. 2013 [Acceso 3 noviembre 2017]; 116 (13). Disponible en: http://www. biomedcentral.com/1471-244X/13/116.

2. Fauman MA. Manual diagnóstico y estadístico de los trastornos mentales. DSM. 5a ed. Washington D.C: MASSON SA. 2003.

3. Pérez B, Fernández A, Bringas $C$, Rodriguez F. Prevalencia de los trastornos de personalidad de acuerdo con los criterios del CIE-10. Estudio en una muestra penitenciaria. Revista de Psicología UVM [internet]. 2014 [Acceso 3 noviembre 2017]; 4 (7): 7-21. Disponible en: http://sitios.uvm.cl/revistapsicologia/revistadetalle.php/7/40/.

4. Ferrer Botero, A. Et al. Prevalencia de los trastornos de la personalidad en estudiantes universitarios de la ciudad de Medellín. Revista de Psicología Universidad de Antioquia. [internet]. 2015 [Acceso 3 noviembre 2017]; 7(1):73-96. Disponible en: http://tesis.udea.edu.co/ dspace/bitstream/10495/7696/1/FerrerAlberto_2015_Pr evalenciaTrastornosPersonalidad.pdf.

5. Rodríguez J, Kohn R, Aguilar S. Epidemiología de los trastornos mentales en América Latina y el Caribe. [internet]. 2009 [Acceso 3 noviembre 2017]. Disponible en: http://iris.paho.org/xmlui/bitstream/ handle/123456789/740/9789275316320.pdf.
6. Colombia. Ministerio de Salud de Colombia. Encuesta Nacional de Salud Mental [internet]. 2015 [Acceso 3 noviembre 2017]. Disponible en: http://www.odc.gov.co/ Portals/1/publicaciones/pdf/consumo/estudios/nacionale s/CO031102015-salud_mental_tomol.pdf.

7. Campo A, Cassiani C. Trastornos mentales más frecuentes: prevalencia y algunos factores sociodemográficos asociados.Rev. Colomb. Psiquiat [internet]. 2008 [Acceso 3 noviembre 2017]. Disponible en: http://www.redalyc.org/pdf/806/80637410.pdf.

8. Maghsoodloo S, Ghodousi A, Karimzadeh T. The relationship of antisocial personality disorder and history of conduct disorder with crime incidence in schizophrenia. $\mathrm{J}$ Res Med Sci. [internet]. 2012 [Acceso 3 noviembre 2017]; 17(6): 566-571. Disponible en: https://www. ncbi.nlm.nih.gov/pmc/articles/PMC3634297/.

9. Barria J. Trastornos de personalidad, psicopatología y nivel de riesgo en una muestra chilena de hombres maltratadores: ocho tipos de maltratadores. Rev. chil. neuro-psiquiatr. [internet]. 2014 [Acceso 3 noviembre 2017]; 52(4). Disponible en: http://www.scielo.cl/ scielo. php? script =sci_arttext\&pid=S 0717 92272014000400006.

10. Hicks B, Vaidyanathan U, Patrick C. Balidating female psychopathy subtypes: Differences in personality antisocial and violent behavior, substance abuse, trauma, and mental health. PMC.[internet]. 2010 [Acceso 4 de Dic 2017]; 1(1): 38-57. Disponible en: https://www. ncbi.nlm.nih.gov/pmc/articles/PMC2889701/.

11. Langley K, Holmans P, Bree M, Thapar A. Effects of low birth weight, maternal smoking in pregnancy and social class on the phenotypic manifestation of Attention Deficit Hyperactivity Disorder and associated antisocial behaviour: investigation in a clinical sample. BMC Psychiatry. [internet]. 2007 [Acceso 4 de Dic 2017]; 7(26). Disponible en: http://www.biomedcentral.com/1471$244 \mathrm{X} / 7 / 26$

12. Hyde L, Hariri A, Manuck S, Votruba A. Amygdala reactivity and negative emotionality: Divergent correlates of antisocial personality and psychopathy traits in a community sample. Journal of Abnormal Psychology. [internet]. 2014 [Acceso 4 noviembre 2017]; 123(1): 214224. Disponible en: https://www.ncbi.nlm.nih.gov/ pubmed/24661171.

13. Chang $\mathrm{H}$. Et al. Possible association between SIRT1 single nucleotide polymorphisms and predisposition to antisocial personality traits in Chinese adolescents. Sci Rep. [internet]. 2017 [Acceso 4 noviembre 2017]; 7(1099) Disponible en: https://www.ncbi.nlm.nih.gov/ pmc/articles/PMC5430697/.

14. Ma G, Fan H, Shen C, Wang W. Genetic and neuroimaging features of personality disorders: State of art. Nuerosci Bull. [internet]. 2016 [Acceso 4 noviembre 2017]; 32(3): 286-306 Disponible en: https://www. ncbi.nlm.nih.gov/pmc/articles/PMC5563771/.

15. Rautiainen M. Et al. Genome-wide association study of antisocial personality disorder. Tsansl Psychiatry. [internet]. 2016 [Acceso 4 noviembre 2017]; 6(9) Disponible en: https://www.ncbi.nlm.nih.gov/pmc/ articles/PMC5048197/.

16. Jorev M, Et al. Temperament and Maltreatment in the Emergence of Borderline and Antisocial Personality Pathology during Early Adolescence. J Can Acad Child and Adolesc Psychiatry. [internet]. 2013 [Acceso 4 
noviembre 2017]; 22(3): 220-9 Disponible en: https://www.ncbi.nlm.nih.gov/pubmed/23970911.

17. Hikal W. Criminología psicoanalítica conductual y del desarrollo [Internet]. 1 Edición. León Mexico 2005. [Acceso 4 noviembre 2017]. Disponible en: http://funvic.org/CriminologiaPsicoanaitica.pdf.

18. Taboada A. La personalidad antisocial. [internet]. 2008 [Acceso 4 de noviembre 2017]; 22: 463-472. Disponible en: http://www.revistacultura.com.pe/revistas/ RCU_22_1_la-personalidad-antisocial.pdf.

19. España. Asociasión Española de Psiquiatria del Niño y el Adolescente (AEPNYA). Trastornos del comportamiento: negativista desafiante y disocia. [internet]. 2008 [Acceso 4 noviembre 2017]. Disponible en: http://www.aeped.es/sites/default/files/ documentos/trastornos del comportamiento negativis ta_desafiante_y_disocial.pdf.

20. Baterman A, O'Connell J, Lorenzini N, Gardner T, Fonagy P. A randomised controlled trial of mentalizationbased treatment versus structured clinical management for patients with comorbid borderline personality disorder and antisocial personality disorder. BMC Psychiatry [internet]. 2016 [Acceso 4 noviembre 2017]; 16(1): 304. Disponible en: https://www.ncbi.nlm.nih.gov/ pmc/articles/PMC5006360/.

21. Kasinathan J, Sharp G, Barker A. Evaluation of olanzapine pamoate depot in seriously violent males with schizophrenia in the community. Ther Adv Psychopharmacol [internet]. 2016 [Acceso 4 noviembre 2017]; 6(5): 301-307. Disponible en: https://www. ncbi.nlm.nih.gov/pmc/articles/PMC5036137

22. Brown D. Et al. Clozapine: an effective treatment for seriously violent and psychopathic men with antisocial personality disorder in a UK high-security hospital. CNS
Spectr [internet]. 2014 [Acceso 4 de noviembre 2017]; 19(5): 391-402. Disponible en: https://www. ncbi.nlm.nih.gov/pmc/articles/PMC4255317/

23. Maghsoodloo S, Ghodousi A, Karimzadeh T. The relationship of antisocial personality disorder and history of conduct disorder with crime incidence in schizophrenia. J es Med Sci [internet]. 2012 [Acceso 4 de noviembre 2017]; 17(6): 566-57. Disponible en: https://www.ncbi.nlm.nih.gov/pmc/articles/PMC363429 71

24. Douglas B, Widige T. Describing Ted Bundy's Personality and Working towards DSM-V. Independent Practitioner. [internet]. 2007 [Acceso 4 noviembre 2017]; 27(1): 20-22. Disponible en: https://pdfs. semanticscholar.org/eb9a/3fbe2d3bbe7fe180e7f161e3 5e4188cd3759.pdf.

25. Fernández J. Luis Alfredo Garavito Cubillo y su marca indeleble. Revista Electrónica de Psicología Social [internet]. 2009 [Acceso 4 noviembre 2017]. Disponible en: http://www.funlam.edu.co/revistas/index.php/ poiesis/article/viewFile/152/141.

26. Esbec E, Echeburúa E. La evaluacion de los trastornos de la personalidad degun el DSM-5: Recursos y limitaciones. Ter Psicol [internet]. 2014 [Acceso 4 de noviembre 2017]; 32(3). Disponible en: http://www. scielo.cl/scielo.php?script=sci_arttext\&pid=S071848082014000300008.

27. Alvarado I, Nieves I, Garcia N. El trastorno antisocial de la personalidad en personas institucionalizadas en Puerto Rico: estudio de casos. Rev Puertorriq Psicol. [internet]. 2014 [Acceso 4 noviembre 2017]; 25. Disponible en: http://pepsic.bvsalud.org/ scielo.php?script=sci_arttext\&pid=S194620262014000100005. 